\title{
Monoclonal Expansion with Integration of High-Risk Type Human Papillomaviruses Is an Initial Step for Cervical Carcinogenesis: Association of Clonal Status and Human Papillomavirus Infection with Clinical Outcome in Cervical Intraepithelial Neoplasia
}

\author{
Yutaka Ueda, Takayuki Enomoto, Takashi Miyatake, Keiichiro Ozaki, \\ Tatsuo Yoshizaki, Hiroyuki Kanao, Yuko Ueno, Ryuichi Nakashima, \\ Kenneth R. Shroyer, and Yuji Murata
}

Department of Obstetrics and Gynecology (YU, TE, TM, KO, TY, HK, YU, RN, YM), Osaka University Faculty of Medicine, Suita, Osaka, Japan; and Department of Pathology (KRS), University of Colorado Health Sciences Center, Denver, Colorado

\begin{abstract}
SUMMARY: To define the natural history of cervical intraepithelial neoplasia (CIN) as related to clonal status, we evaluated 20 cases of CIN1 and 18 cases of CIN2 that had been clinically followed for 7 to 48 months at Osaka University Hospital. These included 10 cases that progressed, 15 cases that persisted, and 13 cases that regressed. We analyzed the clonal status of each case by analysis of the pattern of $X$-chromosomal inactivation. Human papillomavirus (HPV) infection was detected by PCR-RFLP analysis. CINs that are monoclonal or infected by high-risk HPVs are more likely to progress or persist than cases that are polyclonal or negative for high-risk HPVs $(p=0.009$ for monoclonal vs polyclonal, $p=0.024$ for high-risk HPV positive vs negative $p=0.024)$. Eighteen (90\%) of 20 monoclonal, high-risk HPV-associated CINs progressed or persisted, whereas 9 (60\%) of 15 polyclonal or high-risk HPV-negative CINs regressed. Therefore, the combination of clonality status and high-risk type HPV infection was significantly correlated with clinical outcome $(p=0.003)$. The physical status of the HPV genome was evaluated in 17 cases of HPV-16 positive CINs by real-time PCR. Of those, the HPV viral genome was present in both episomal and integrated forms in $14 \mathrm{CINs}(84 \%)$, and 12 of these cases (86\%) were monoclonal in composition. By contrast, all three CINs in which the HPV genome was present in episomal form were polyclonal. In one CIN1 that was polyclonal, HPV-16 was originally present in episomal form but after 24 months, the patient developed a monoclonal CIN3 in which the HPV-16 genome was present in mixed form. These results may imply that HPV viral integration into the host genomic DNA is associated with progression from polyclonal to monoclonal status in CIN. These events may play a fundamental role in the progression from low-grade to higher grade dysplasia of the cervical mucosa. (Lab Invest 2003, 83:1517-1527).
\end{abstract}

C ervical intraepithelial neoplasia $(\mathrm{CIN})$ is regarded as a precursor of squamous cell carcinoma of the uterine cervix, and infection of high-risk human papillomavirus (HPV) is thought to be an important initiating factor of cervical carcinogenesis (Iwasaka et al, 1994; Noda et al, 1976). Previous studies demonstrated that high-risk HPVs were present in $85 \%$ and $92 \%$ of CIN2 and CIN3, respectively (Bergeron et al, 1992), and that CINs infected with high-risk HPVs such as type 16 have a significantly higher relative risk of progression than CINs that are negative for highrisk HPVs (Kataja et al, 1992). In cervical carcinomas, the viral genome of high-risk HPVs is usually inte-

DOI: 10.1097/01.LAB.0000092234.68751.83

Received August 5, 2003.

Address reprint requests to: Dr. T. Enomoto, Department of Obstetrics and Gynecology, Osaka University Faculty of Medicine, 2-2, Yamadaoka, Suita, Osaka 565-0871, Japan.E-mail: enomoto@gyne.med.osaka-u.ac.jp grated into the host genome. In the process of integration of HPV, some part of the E2 open reading frame (ORF) is deleted (Das et al, 1992; Kalantari et al, 1998; zur Hausen, 1991). The E2 gene encodes a 48-kDa phosphorylated protein involved in the regulation of viral DNA transcription and replication. Deletion of the E2 ORF up-regulates the oncogenic E6 and E7 genes, resulting in tumor progression (Kalantari et al, 1998). Integration of HPV into the host genome has been demonstrated by 2-dimensional gel electrophoresis, and recently, by quantitatively comparing the levels of E2 and E6 DNA by real-time PCR. An earlier study using 2-dimensional gel electrophoresis showed that integration of high-risk HPV was characteristic of cervical carcinoma but not of CIN (Das et al, 1992). However, recent studies using real-time PCR suggested that integration of high-risk HPV into the host genome occurs in a fraction of cases of $\mathrm{CIN}$ (Nagao et al, 2002; Peitsaro et al, 2002). The factors that influence the HPV-mediated progression of dys- 
plastic lesions to invasive carcinoma have not been determined.

A fundamental principle held by most students of tumor biology is the belief that neoplastic tissues are composed of clonal cell populations (Fialkow, 1976). Although many methods have been used to investigate the clonal composition of tissues, the most consistently informative marker is the cellular pattern of $X$ chromosome inactivation (Enomoto et al, 1994). During embryogenesis in the female, either the paternally derived or the maternally derived $X$ chromosome in each cell is randomly inactivated by methylation of cytosine residues. Once made, the choice is stable through subsequent cycles of cell division (Mandel et al, 1992; Riggs and Pfeifer, 1992). Thus, normal tissues of females show a random pattern of $X$ chromosome inactivation. By contrast, neoplastic tissues from female subjects display a uniform pattern of $X$ chromosome inactivation, consistent with monoclonal composition.

We previously demonstrated monoclonal composition of CIN3s by analyzing the methylation pattern of the human $X$-linked androgen receptor gene (HUMARA) (Enomoto et al, 1997). Although clonal status is an important biomarker of cervical neoplasia, it is still unclear whether the clinical outcome of CINs is related to either clonality status or high-risk HPV status. Furthermore, the association between clonality status and HPV DNA integration into the host genome has not been determined. In the present study, we collected cases of CIN1 and CIN2 that had been clinically followed at the Osaka University Hospital. These included 10 cases in which lesions progressed, 15 cases in which lesions remained persistent, and 13 cases in which lesions regressed. We analyzed the clonal status, the presence of HPV infection, and the physical status of HPV and associated these characteristics with clinical outcome.

\section{Results}

\section{Clonal Analysis}

PCR amplification was performed to generate fragments surrounding the highly polymorphic CAG repeat in exon 1 of the HUMARA gene, using DNA extracted from microdissected tissues as templates. PCR amplification of undigested DNA resulted in the detection of two major HUMARA amplification products of almost identical height in 35 of $38 \mathrm{CIN} 1 / 2$ s. The remaining three cases appeared homozygous for the HUMARA target and, as a result, were excluded from clonality analysis.

Pretreatment of genomic DNA with the methylationsensitive restriction endonuclease Hhal blocked PCR amplification of the unmethylated (active) HUMARA allele. Blocked amplification of one of the two HUMARA targets by Hhal pretreatment of the genomic DNA was interpreted as evidence of a uniform pattern of $X$ chromosome inactivation, consistent with a monoclonal cell population. By contrast, if Hhal pretreatment of the genomic DNA failed to block amplifi- cation of either band, the case was considered to be polyclonal (Allen et al, 1992; Enomoto et al, 1997) (Fig. 1). Of 35 informative cases, 12 (62\%) of 19 CIN1s and $12(75 \%)$ of 16 CIN2s were monoclonal in composition at the initial diagnosis (Table 1).

\section{Detection of HPV}

Testing for HPV DNA was performed by PCR-RFLP analysis in all of the 38 cases. High-risk HPVs were detected in $14(70 \%)$ cases of CIN1 and 15 (83\%) cases of CIN2, respectively, in total. Of $14 \mathrm{CIN} 1 \mathrm{~s}$ positive for high-risk HPV, HPV-16 was detected in 8 cases (57\%), HPV-52 in 2 cases (14\%), HPV-58 in 2 cases (14\%), HPV-18 in a single case $(7 \%)$, and HPV-31 in another case (7\%). Of 15 CIN2s positive for high-risk HPV, HPV-16 was detected in 9 cases (60\%), HPV-52 in 2 cases (13\%), HPV-58 in a single case (7\%), and HPV-33 in another case (7\%). HPV typing was undetermined in two cases (13\%) of CIN2. Lowrisk HPVs were detected in three cases $(21 \%)$ of CIN1 and two cases (13\%) of CIN2, and all of which were HPV-11. Coinfection by multiple HPV types was detected in a single case $(7 \%)$ of CIN1 and in four cases (37\%) of CIN2, including detection of both HPV-11 and HPV-16 in two cases, HPV-16 and HPV-52 in two cases, and HPV-11 and HPV-33 in one case.

The association of HPV infection with clonal status of CIN1/2 was evaluated. Monoclonal composition was observed in $10(71 \%)$ of 14 cases of CIN1 and in $10(83 \%)$ of 12 cases of CIN2 that tested positive for high-risk HPVs and informative for clonality analysis. By contrast, monoclonal composition was found in only two (40\%) of five cases with CIN1 and two (50\%) of four cases with CIN2 that were negative for highrisk HPVs and informative for clonality analysis. Overall, $20(77 \%)$ of $26 \mathrm{CIN} 1 / 2 \mathrm{~s}$ that were positive for high-risk HPV were monoclonal, and 5 (56\%) of 9 CIN1/2s that were negative for high-risk HPVs were polyclonal. Although high-risk HPV-positive CINs tended to be monoclonal and high-risk HPV-negative $\mathrm{CINs}$ tended to be polyclonal, the differences in clonality status compared with HPV test results were not statistically significant $(p=0.07)$.

\section{HPV Physical Status}

The physical status of HPV-16 in CINs was examined by real-time PCR amplification of the E2 and E6 ORFs. Primers were designed to amplify the E2 hinge region, which is usually deleted upon viral integration into the host genome in cervical carcinoma (Kalantari et al, 1998). The detection of both $E 2$ and $E 6$ at equivalent levels was interpreted as an indication that HPV remained in episomal form [Fig. 2, (2)]. The detection of E6 but not E2 was interpreted as evidence of complete HPV integration into the host genome. By contrast, the detection of E6 at relatively high levels with low-level amplification of E2 was interpreted as an indication that the HPV viral genome was present in both integrated and episomal forms [Fig. 2, (3)] (Nagao et al, 2002; Peitsaro et al, 2002). 


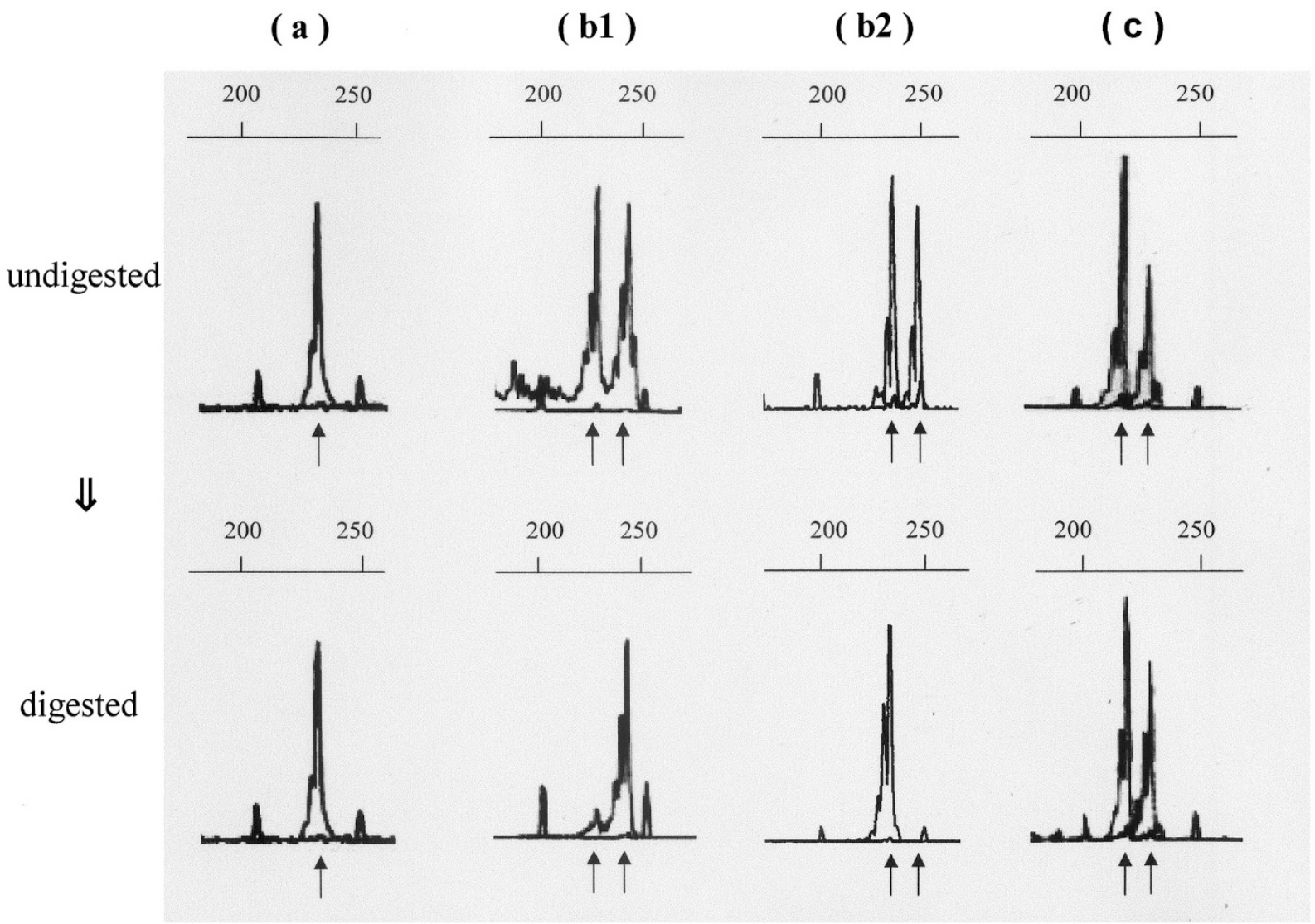

Figure 1.

Clonality analysis of cervical intraepithelial neoplasia (CIN) by a PCR-based method (representative cases). Gene Scan imaging of amplification products. Genomic DNA derived was digested with the methylation-sensitive restriction endonuclease Hhal, and PCR amplification was performed, targeting a highly polymorphic CAG repeat in exon 1 of the HUMARA gene. (a), Not informative case. PCR of either undigested or digested genomic DNA resulted in amplification of a single major HUMARA peak. This case was homozygous in the HUMARA gene locus and was not informative for clonality analysis. (b1) and (b2), Monoclonal A and monoclonal B. Informative cases showing evidence of monoclonal composition. PCR of undigested DNA resulted in amplification of two major HUMARA peaks from each case. Pretreatment of genomic DNA in each case with Hhal, followed by PCR blocked amplification of the larger (monoclonal A) or smaller (monoclonal B) one of the two peaks, indicating a uniform pattern of $X$ chromosome inactivation, consistent with the presence of a clonal cell population. (c), Informative cases showing evidence of polyclonal composition. PCR of undigested or digested DNA resulted in amplification of two major peaks, consistent with a polyclonal cell population.

When pZE67 was used as PCR template, amplification of E6 but not E2 was observed, which served as a control for the integrated form of HPV. PCR amplification of E6 but not E2 was observed in three cases of cervical carcinoma, consistent with HPV integration into the host genome [Fig. 2, (1)]. The ratio of E2 to E6 amplification was 0 to $<0.5$ (range $0.02-0.33$ ) in five of eight CIN1s and in all nine CIN2s that were positive for HPV-16, consistent with the presence of HPV-16 DNA in both episomal and integrated forms. The ratio of E2 to E6 was $>0.5$ (range $0.68-0.92$ ) in three CIN1s, indicating that the viral genome was not integrated but remained in episomal form in those cases. One case of polyclonal CIN1 (Case 1) with the episomal form of HPV-16 progressed to a monoclonal CIN3 with both integrated and episomal forms of HPV-16 (Fig. 3).

\section{Association of HPV Genomic Status with Clonality}

The physical status of the HPV genome was compared with the results of clonality analysis in CINs. HPV-16 was present in both integrated and episomal forms in all 12 monoclonal CIN1/2s. HPV-16 was present in only the episomal form in three of five cases of polyclonal CIN1/2s and as a mixed form in the remaining two polyclonal cases. The association between clonal status and HPV physical status of CIN1/2 was significant $(p=0.015)$.

\section{Association of Clonality with Clinical Outcome of CIN}

Monoclonal CIN1/2s were more likely to progress or remain persistent than polyclonal $\mathrm{CIN} 1 / 2 \mathrm{~s}$, and polyclonal lesions were more likely to regress than monoclonal lesions. Of $19 \mathrm{CIN} 1 \mathrm{~s}$ that were informative for clonal analysis, 12 cases were monoclonal at the initial diagnosis. Of those, four cases progressed to $\mathrm{CIN} 2 / 3$ within 7 to 18 months, whereas lesions remained persistent for 8 to 36 months in six cases and lesions regressed within 3 to 6 months in two cases. In most of these cases, clonal status, HPV infection, and HPV physical status were examined on all biopsy specimens that were obtained at 3- to 6-month intervals. The results of clonal status, HPV infection, and HPV physical status were consistent throughout the observation period in most of the cases, except in Cases 11, 13, 
Table 1. Clonality and HPV Infection of CIN

\begin{tabular}{|c|c|c|c|c|c|}
\hline Case & Months & Histologic examination & Clonality & HPV type & HPV-16 physical status \\
\hline \multicolumn{6}{|c|}{ Progressed } \\
\hline \multirow[t]{2}{*}{1} & 0 & CIN1 & Poly & 16 & Episomal \\
\hline & 24 & CIN3 & Mono A & 16 & Mixed \\
\hline \multirow[t]{2}{*}{2} & 0 & CIN1 & Mono B & 18 & $\mathrm{NI}$ \\
\hline & 15 & CIN3 & Mono B & 18 & $\mathrm{NI}$ \\
\hline \multirow[t]{2}{*}{3} & 0 & CIN1 & Mono B & 52 & $\mathrm{NI}$ \\
\hline & 8 & CIN2 & Mono B & 52 & $\mathrm{NI}$ \\
\hline \multirow[t]{2}{*}{4} & 0 & CIN1 & Mono B & 52 & $\mathrm{NI}$ \\
\hline & 7 & CIN3 & Mono B & 52 & $\mathrm{NI}$ \\
\hline \multirow[t]{2}{*}{5} & 0 & CIN1 & Mono A & 16 & Mixed \\
\hline & 18 & CIN3 & Mono A & 16 & Mixed \\
\hline \multirow[t]{2}{*}{6} & 0 & CIN2 & Mono A & 16 & Mixed \\
\hline & 30 & CIN3 & Mono A & 16 & Mixed \\
\hline \multirow[t]{2}{*}{7} & 0 & CIN2 & Mono B & 16 & Mixed \\
\hline & 14 & CIN3 & Mono B & 16 & Mixed \\
\hline \multirow[t]{2}{*}{8} & 0 & CIN2 & Mono B & 16 & Mixed \\
\hline & 20 & CIN3 & Mono B & 16 & Mixed \\
\hline \multirow[t]{2}{*}{9} & 0 & CIN2 & Mono B & + & $\mathrm{NI}$ \\
\hline & 9 & CIN3 & Mono B & + & $\mathrm{NI}$ \\
\hline \multirow[t]{2}{*}{10} & 0 & CIN2 & Mono A & 11,16 & Mixed \\
\hline & 20 & CIN3 & Mono A & 11,16 & Mixed \\
\hline \multicolumn{6}{|c|}{ Persistent } \\
\hline \multirow[t]{2}{*}{11} & 0 & CIN1 & Mono A & 16 & Mixed \\
\hline & 39 & CIN1 & Poly & 52 & $\mathrm{NI}$ \\
\hline \multirow[t]{2}{*}{12} & 0 & CIN1 & Poly & 16 & Episomal \\
\hline & 32 & CIN1 & Poly & 16 & Episomal \\
\hline \multirow[t]{3}{*}{13} & 0 & CIN1 & Mono B & - & $\mathrm{NI}$ \\
\hline & 9 & CIN1 & Mono A & - & $\mathrm{NI}$ \\
\hline & 22 & CIN1 & Poly & - & $\mathrm{NI}$ \\
\hline \multirow[t]{2}{*}{14} & 0 & CIN1 & Mono A & 31 & $\mathrm{NI}$ \\
\hline & 27 & CIN1 & Mono A & 31 & $\mathrm{NI}$ \\
\hline \multirow[t]{2}{*}{15} & 0 & CIN1 & Poly & - & $\mathrm{NI}$ \\
\hline & 9 & CIN1 & Poly & - & $\mathrm{NI}$ \\
\hline \multirow[t]{2}{*}{16} & 0 & CIN1 & Not informative & - & $\mathrm{NI}$ \\
\hline & 16 & CIN1 & Not informative & - & $\mathrm{NI}$ \\
\hline 17 & 0 & CIN1 & Mono B & 58 & $\mathrm{NI}$ \\
\hline & 11 & CIN1 & Mono B & 58 & $\mathrm{NI}$ \\
\hline 18 & 0 & CIN1 & Mono B & 58 & $\mathrm{NI}$ \\
\hline & 22 & CIN1 & Mono B & 58 & $\mathrm{NI}$ \\
\hline & 25 & CIN1 & Mono A & - & $\mathrm{NI}$ \\
\hline & 36 & CIN1 & Mono A & - & $\mathrm{NI}$ \\
\hline 19 & 0 & CIN1 & Poly & 16 & Mixed \\
\hline & 20 & CIN1 & Poly & 16 & Mixed \\
\hline & 29 & CIN1 & Mono A & 16 & Mixed \\
\hline 20 & 0 & CIN1 & Mono A & 11,16 & Mixed \\
\hline & 8 & CIN1 & Mono A & 11,16 & Mixed \\
\hline 21 & 0 & CIN2 & Mono A & 16 & Mixed \\
\hline & 15 & CIN2 & Mono A & 16 & Mixed \\
\hline 22 & 0 & CIN2 & Mono B & 16,52 & Mixed \\
\hline & 18 & CIN2 & Mono B & 16,52 & Mixed \\
\hline 23 & 0 & CIN2 & Mono A & - & $\mathrm{NI}$ \\
\hline & 47 & CIN2 & Mono A & - & $\mathrm{NI}$ \\
\hline 24 & 0 & CIN2 & Mono B & 16,52 & Mixed \\
\hline & 30 & CIN2 & Mono B & 16,52 & Mixed \\
\hline 25 & 0 & CIN2 & Mono A & 11,33 & $\mathrm{NI}$ \\
\hline & 13 & CIN2 & Mono A & 11,33 & $\mathrm{NI}$ \\
\hline
\end{tabular}


Table 1. Continued

\begin{tabular}{|c|c|c|c|c|c|}
\hline Case & Months & Histologic examination & Clonality & HPV type & HPV-16 physical status \\
\hline \multicolumn{6}{|c|}{ regressed } \\
\hline \multirow[t]{2}{*}{26} & 0 & CIN1 & Poly & 16 & Episomal \\
\hline & 30 & No abnormal cytology & & & \\
\hline \multirow[t]{2}{*}{27} & 0 & CIN1 & Mono A & 16 & Mixed \\
\hline & 35 & No abnormal cytology & & & \\
\hline \multirow[t]{2}{*}{28} & 0 & CIN1 & Poly & 11 & $\mathrm{NI}$ \\
\hline & 41 & No abnormal cytology & & & \\
\hline \multirow[t]{2}{*}{29} & 0 & CIN1 & Mono B & - & $\mathrm{NI}$ \\
\hline & 30 & No abnormal cytology & & & \\
\hline \multirow[t]{2}{*}{30} & 0 & CIN1 & Poly & 11 & $\mathrm{NI}$ \\
\hline & 29 & No abnormal cytology & & & \\
\hline \multirow[t]{2}{*}{31} & 0 & CIN2 & Not informative & - & $\mathrm{NI}$ \\
\hline & 42 & No abnormal cytology & & & \\
\hline \multirow[t]{2}{*}{32} & 0 & CIN2 & Mono A & - & $\mathrm{NI}$ \\
\hline & 41 & No abnormal cytology & & & \\
\hline \multirow[t]{2}{*}{33} & 0 & CIN2 & Poly & - & $\mathrm{NI}$ \\
\hline & 30 & No abnormal cytology & & & \\
\hline \multirow{2}{*}{34} & 0 & CIN2 & Poly & - & $\mathrm{NI}$ \\
\hline & 30 & No abnormal cytology & & & \\
\hline \multirow[t]{2}{*}{35} & 0 & CIN2 & Mono A & 16 & Mixed \\
\hline & 17 & No abnormal cytology & & & \\
\hline \multirow[t]{2}{*}{36} & 0 & CIN2 & Not informative & + & $\mathrm{NI}$ \\
\hline & 16 & No abnormal cytology & & & \\
\hline \multirow[t]{2}{*}{37} & 0 & CIN2 & Poly & 58 & $\mathrm{NI}$ \\
\hline & 20 & No abnormal cytology & & & \\
\hline \multirow[t]{2}{*}{38} & 0 & CIN2 & Poly & 16 & Mixed \\
\hline & 15 & No abnormal cytology & & & \\
\hline
\end{tabular}

Months, months after initial diagnosis; Mono A, monoclonal with allele of lower-molecular-weight inactivation; Mono B, monoclonal with allele of higher-molecular-weight inactivation; Poly, polyclonal; +, positive for high-risk HPV but type not determined; -, HPV not detected; Episomal, episomal form; Mixed, mixed type of episomal form and integrated form; NI, not informative for HPV 16 physical status (HPV positive but not type 16).

18, and 19. Cases 11 and 19, which were monoclonal CIN1s at the initial diagnosis, converted to polyclonal CIN1s 39 months and 29 months later, respectively. Cases 13 and 18, which were also monoclonal CIN1s at the initial diagnosis, persisted histologically but converted to the opposite pattern of $X$ chromosome inactivation after 9 months and 25 months, respectively, suggesting that the original lesions did not persistent but that additional lesions developed de novo. Furthermore, Case 13 showed evidence of regression of the second monoclonal CIN I but developed a polyclonal CIN I at 22 months after the initial diagnosis. Of seven polyclonal CIN1s, one case progressed to a monoclonal CIN3 (24 months of observation), three cases persisted (9-32 months of observation), and three cases regressed (3-6 months of observation). Of 16 informative CIN2s, 12 cases were monoclonal at the initial diagnosis. Of those, progression to CIN3 was seen within 9 to 30 months in five cases, five cases persisted during 13 to 47 months, and two cases regressed. All four polyclonal CIN2s regressed within 3 to 6 months after initial diagnosis. In total, 20 (83\%) of 24 monoclonal CIN1/2 progressed or persisted, whereas 7 (64\%) of 11 polyclonal CIN1/2 regressed. Therefore, the association between clonal status and clinical outcome for CIN $1 / 2$ was significant $(p=0.009)$ (Table 2).

\section{Association of High-Risk HPV Infection with Clinical Outcome of CIN}

Cases of CIN1/2 that contained high-risk HPVs were more likely to progress or persist than cases that were negative for high-risk HPVs, and CIN1/2s that were negative for high-risk HPVs were more likely to regress than cases that tested positive for high-risk HPVs. Among $14 \mathrm{CIN} 1 \mathrm{~s}$ that tested positive for high-risk HPVs, 5 cases progressed to higher grade lesions (7-24 months of observation), 7 cases persisted (8-39 months of observation), and 2 cases regressed (3-6 months of observation). Of six CIN1s that were negative for high-risk HPVs, three cases persisted during a 9- to 22-month period of observation, but three cases regressed within 3 to 6 months. Of $13 \mathrm{CIN} 2 \mathrm{~s}$ that contained high-risk HPVs, 5 cases progressed within 9 to 30 months and 4 cases persisted during 13 to 47 months, whereas 4 cases regressed within 3 to 6 months. Of five CIN2s that were negative for high-risk HPVs, four cases regressed, but a single case persisted. In summary, 21 (78\%) of $27 \mathrm{CIN} 1 / 2 \mathrm{~s}$ that were positive for high-risk HPVs progressed or persisted, but $4(36 \%)$ of 11 $\mathrm{CIN} 1 / 2 \mathrm{~s}$ that were negative for high-risk HPVs progressed or persisted. The association of high-risk HPV infection with clinical outcome of CIN was significant $(p=0.024)$ (Table 2). 


\section{(1)}

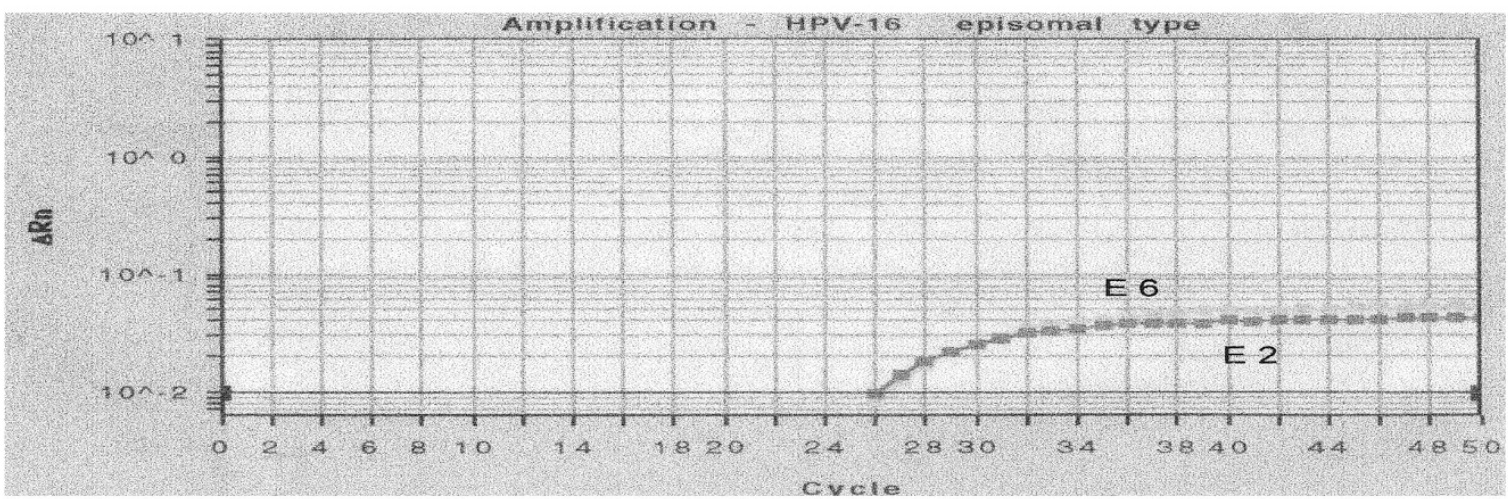

(2)

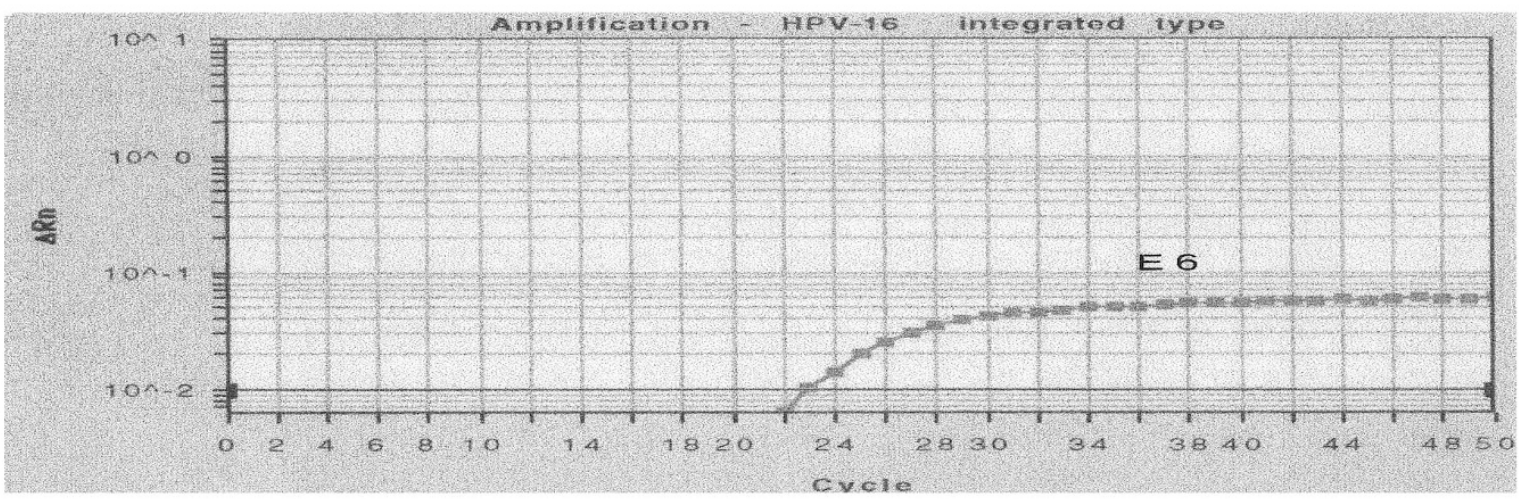

(3)

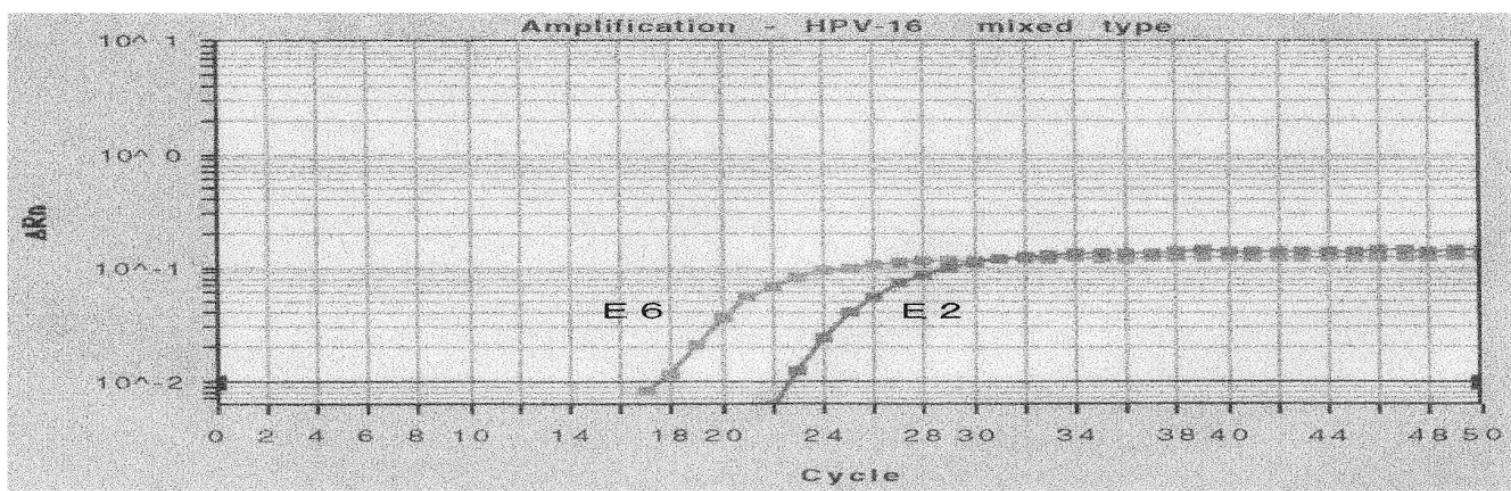

Figure 2.

Examples of real-time PCR analysis of three types of human papillomavirus (HPV) physical status. Run profiles of $\Delta \mathrm{Rn}$ (normalized fluorescence emission subtracted baseline fluorescence) versus PCR cycles in three types are shown. (1) Episomal type: equivalent copy numbers of both E2 and E6 were detected. (2) Integrated type: E6 amplification without E2 amplification was detected. (3) Mixed type: copy numbers of E2 were smaller than E6 but still present.

\section{Association of Clonal Status and High-Risk HPV Infection with Clinical Outcome of CIN}

Monoclonal CIN1/2s that were positive for high-risk HPV were more likely to progress or persist than CIN1/2s that were either polyclonal or negative for high-risk HPVs. Of 10 monoclonal CIN1s that tested positive for high-risk HPVs, 4 cases progressed (7-18 month of observation), 5 cases persisted (8-39 month of observation), and a single case regressed within 3 months. Of nine polyclonal or high-risk HPV negative CIN1s, a single case progressed (24 month of observation) and four cases persisted (9-29 month of observation), and four cases regressed within 3 to 6 months. Of 10 monoclonal CIN2s that were positive for high-risk HPVs, 5 cases progressed (9-30 month of observation), 4 cases persisted (13-30 month of 
initial

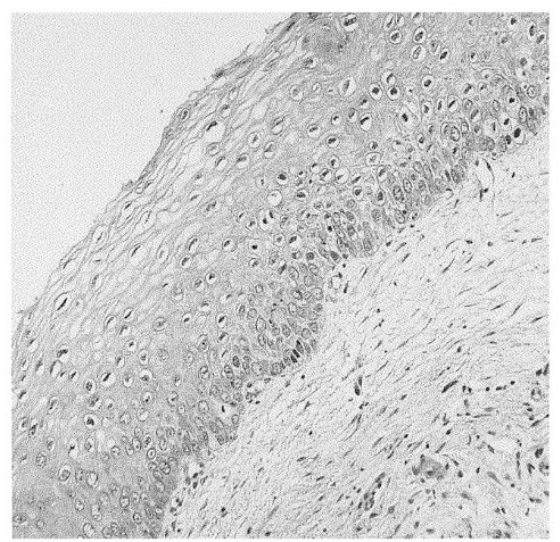

\section{4 months later}

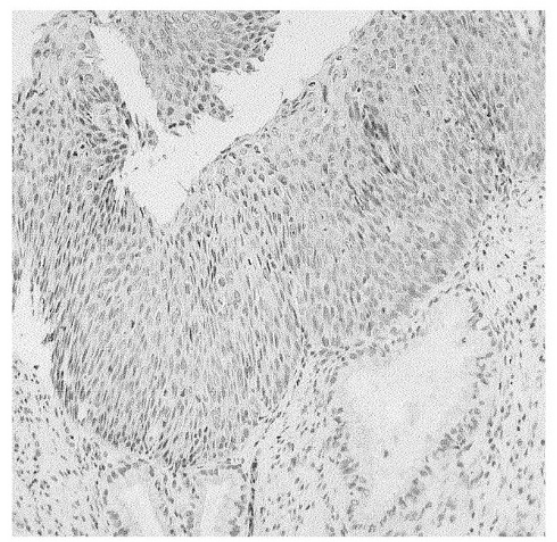

\section{$<\operatorname{H\& E}($ x 100$)>$}

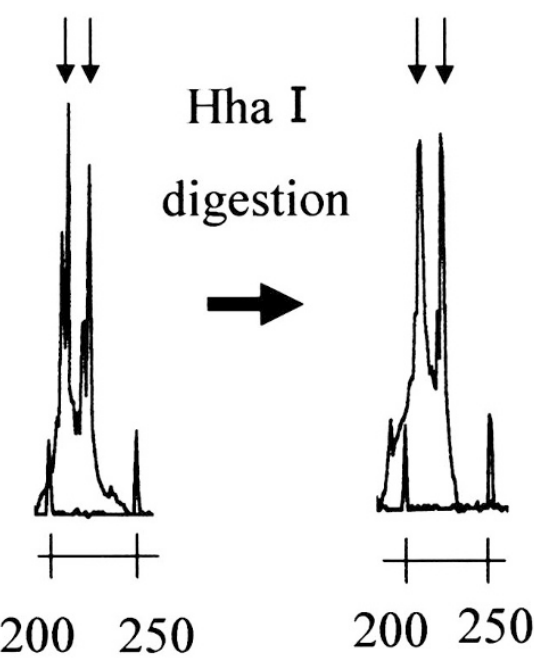

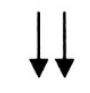

Hha I

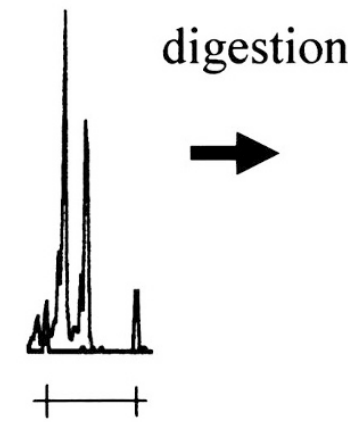

200250
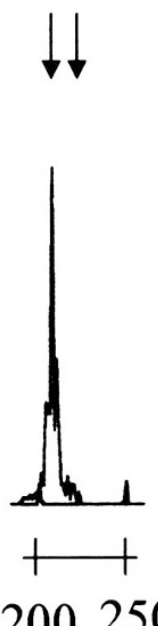

\section{$<$ clonal status $>$}
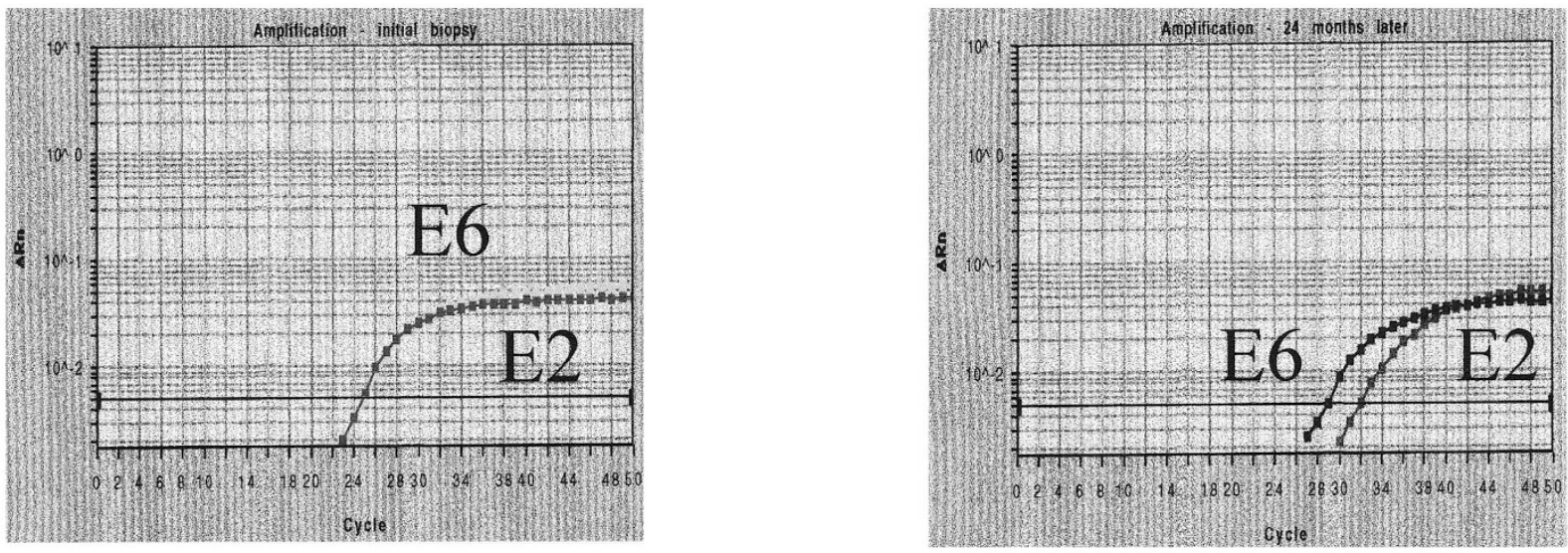

\section{$<$ HPV-16 physical status $>$}

Figure 3.

Change in clonality and HPV-16 physical status in a case of progression of CIN (Case 1). Initial CIN1 lesion was polyclonal in composition and contained HPV-16 in episomal form. Twenty-four months later, this lesion progressed to CIN3, which was monoclonal and contained HPV-16 in the mixed type of integrated and episomal forms. 
Table 2. Association of Clonal Status and High-Risk HPV Infection with Clinical Outcome

\begin{tabular}{lrcc}
\hline & Progressed or persistent & Regressed & $p$ value \\
\hline Clonal status & & & \\
$\quad$ Monoclonal & $20 / 24(83 \%)$ & $4 / 24(17 \%)$ & 0.009 \\
$\quad$ Polyclonal & $4 / 11(36 \%)$ & $7 / 11(64 \%)$ & \\
High-risk HPV infection & & \\
$\quad$ High-risk HPV positive & $21 / 27(78 \%)$ & $6 / 27(22 \%)$ & 0.024 \\
$\quad$ High-risk HPV negative & $4 / 11(36 \%)$ & $7 / 11(64 \%)$ & \\
Clonal status and HPV infection & & & \\
$\quad$ Monoclonal and high-risk HPV positive & $18 / 20(90 \%)$ & $2 / 20(10 \%)$ & 0.003 \\
$\quad$ Other informative cases & $6 / 15(40 \%)$ & $9 / 15(60 \%)$ & \\
\hline
\end{tabular}

observation), and a single case regressed within 3 months. Of six polyclonal or high-risk HPV negative CIN2s, a single case persisted (47 month of observation) and five cases regressed within 3 to 6 months. In total, $18(90 \%)$ of 20 monoclonal $\mathrm{CIN} 1 / 2$ s that were positive for high-risk HPVs progressed or persisted but $9(60 \%)$ of $15 \mathrm{CIN} 1 / 2 \mathrm{~s}$ that were either polyclonal or high-risk HPV negative regressed. The combination of clonality and high-risk HPV infection was thus significantly associated with the clinical outcome of CIN $(p=0.003)$ (Table 2).

\section{Discussion}

$\mathrm{CIN}$ is regarded as a precursor of cervical carcinoma (Noda et al, 1976). Without treatment, a significant proportion of cases of CIN1 and CIN2 will progress to higher grade CIN (Iwasaka et al, 1994; Kataja et al, 1992; Murthy et al, 1990, 1996; Nasiell et al, 1983, 1986). At Osaka University Hospital, 88 cases of CIN1/2s were followed up for 6 to 48 months from 1997 to 2001 . Six (10\%) of 59 CIN1s and 7 (24\%) of 29 progressed to higher grade lesions during the observation period, consistent with previous reports. It has also been reported that CINs that are associated with high-risk HPVs are more likely to progress (Kataja et al, 1992). On the other hand, the correlation between clinical outcome and clonality status of CINs remains relatively unexplored. Previous studies in our laboratory demonstrated monoclonal composition in all 30 $(100 \%)$ of CIN3s, but a single case of CIN2 was polyclonal (Enomoto et al, 1997). Similarly, Park et al (1996) showed that 54 (68\%) of 79 low-grade dysplastic lesions were monoclonal, but that high-grade dysplasia was uniformly monoclonal. In the present study we showed that $62 \%$ of CIN1s and $75 \%$ of CIN2s were monoclonal. However, the proportion of monoclonal test results may not be representative of the general population in Japan because there was case selection bias in the present study. Our cases included $25 \mathrm{CIN} 1 / 2 \mathrm{~s}$ that persisted or progressed at the same area of the cervical mucosa throughout the observation period and $13 \mathrm{CIN} 1 / 2 \mathrm{~s}$ that regressed from 1997 to 2001. Thus our study could be biased by underrepresentation of cases that would normally regress. Even in those cases of CIN1/2 that regressed, however, monoclonal composition of the lesion was dem- onstrated in $4(36 \%)$ of 11 cases and monoclonal composition was demonstrated in approximately $80 \%$ of CIN 1/2s that progressed or persisted. These findings suggest that approximately $40 \%$ to $80 \%$ of CIN1/2 in the general Japanese population could be monoclonal in composition.

The current study demonstrated that the clonality status was significantly associated with the clinical outcome of the $\mathrm{CIN}$, including progression, persistence, or regression. Although a fundamental characteristic of neoplasia is the monoclonal composition of the tissue, $17 \%$ of monoclonal CIN1/2s regressed within 6 months after initial diagnostic punch biopsy. Thus, monoclonal composition was not a uniformly predictive marker of progression. However, it is likely that the natural history of some lesions was altered by either complete excision by punch biopsy or that the associated inflammatory or reparative processes that followed tissue biopsy could have promoted the regression of lesional epithelium. Consistent with these hypotheses, a previous study found that CIN diagnosed by cytology with biopsy regressed significantly more frequently than $\mathrm{CIN}$ diagnosed by cytology without biopsy (Nasiell et al, 1983). As a further alternative explanation, some monoclonal lesions may be removed by the immune system of the host.

The current investigation also demonstrated that high-risk HPV infection was significantly associated with the clinical outcome of CIN. This result is concordant with a previous study that found that $34 \%$ of $\mathrm{CINs}$ that were positive for high-risk HPVs progressed and that $44 \%$ regressed. By contrast, only $11 \%$ of CINs that were negative for high-risk HPVs but were positive for low-risk HPVs progressed and $62 \%$ of those regressed (Kataja et al, 1992).

To date, there are no previous reports that have described the correlation of clinical outcome of CIN with both clonality status and with the detection of high-risk HPVs. The current study demonstrated for the first time that both clonal status and the presence of high-risk HPVs are significantly associated with the clinical outcome. Furthermore, both clonal status and high-risk HPV infection were much better predictors for clinical outcome.

Earlier studies using 2-dimensional gel electrophoresis showed that high-risk HPV integration was present in most cases of cervical carcinoma but was 
rarely present in CIN (Das et al, 1992). In recent studies using real-time PCR (Nagao et al, 2002; Peitsaro et al, 2002), the integration of the genome of high-risk HPVs was detected not only in cervical carcinomas but also in CINs. Peitsaro et al (2002) showed that 14 of 15 HPV-16-positive CIN1/2s contained HPV-16 DNA in either integrated or mixed form. Our results regarding the status of HPV-16 DNA in CIN1 and CIN2 is concordant with the previous reports. The current study provides additional important information regarding the association between HPV genomic integration and clonality status. Our observation that one case of polyclonal CIN1 (Case 1) with episomal HPV-16 progressed to a monoclonal CIN3 with both episomal and integrated HPV-16 implies that the integration of high-risk HPV may trigger the emergence of a monoclonal cell population, as an important step in cervical carcinogenesis. Further studies will be necessary, however, to determine whether HPV genomic integration is the initiating event for clonal expansion or whether viral integration represents an epiphenomenon associated with other processes that are etiologic for cervical neoplasia. Productive infections of the cervical mucosa support the proliferation of the HPV genome in episomal form and represent polyclonal proliferative processes. The integration of high-risk HPV DNA into the host genome can result in overexpression of the E6 and E7 oncoproteins, by disruption of the normal regulatory effects of the E2 ORF (Das et al, 1992; Kalantari et al, 1998; zur Hausen, 1991). The overexpression of E6 and E7 disrupts normal p53 and pRb/E2F cell cycle regulatory mechanisms, which could lead to the emergence of clonal cell populations.

Although the clinical outcome of CINs cannot be predicted on the basis of histologic assessment, the analysis of clonality and the detection of high-risk HPV genomic integration, either alone or in combination, might help identify cases with more aggressive biologic potential. Further larger scale prospective studies are indicated to confirm the current findings and to determine whether analysis of clonality or HPV physical status could play a prognostic role to help guide the clinical management of patients with potentially premalignant lesions of the cervical mucosa.

\section{Materials and Methods}

\section{Materials}

From 1997 to 2001, a total of 107 new cases of CIN1/2 were registered at the Osaka University Hospital, Osaka, Japan. Eighty-eight cases had clinical follow-up of more than 6 months. Initial diagnoses were made by cervical smear and punch biopsy under colposcopic examination. During the follow-up period, cervical smears were taken and lesions were carefully monitored by colposcopy every 1 to 3 months for CIN2 and every 3 to 6 months for CIN1. Colposcopic examinations with punch biopsies were performed for histologic confirmation of visible lesions. Of 59 CIN1s, 6 cases (10\%) progressed to CIN2/3 (median time to progression: 16.5 months, range 7-24 months), 13 cases (22\%) persisted (median time of follow-up: 19.0 months, range 8-36 months), whereas lesions regressed in 40 cases (68\%). Of 29 CIN2s, 7 cases (24\%) progressed to CIN3 (median time to progression: 14.0 months, range 9-30 months), and another 7 cases (24\%) persisted (median time of follow-up: 18.0 months, range 13-47 months), whereas lesions regressed within 6 months in 15 cases (52\%), as confirmed by normal cytology results every 3 to 6 months after regression. Therapeutic cone biopsies were performed when lesions progressed to CIN3. Of 19 CIN1s and $14 \mathrm{CIN} 2 \mathrm{~s}$ that progressed or persisted, lesions remained localized to the same area of the uterine cervix throughout the observation period in $15 \mathrm{CIN} 1 \mathrm{~s}$ (79\%) and 10 CIN2s (71\%). These 25 cases were selected for the clonality analysis and the analysis of HPV genomic status. In addition, 5 of $40 \mathrm{CIN} 1 \mathrm{~s}$ and 8 of $15 \mathrm{CIN} 2 \mathrm{~s}$ that regressed were randomly selected and included in the study. In total, 20 CIN1s and 18 CIN2s were analyzed (median duration of follow-up: 28.0 months, range $7-48$ months). Of the 20 CIN1s, 4 cases progressed to CIN3 within 7 to 24 months; a single case progressed to CIN2 within 8 months. Lesions persisted in $10 \mathrm{CIN} 1 \mathrm{~s}$ for 8 to 39 months and regressed within 3 to 6 months in 5 CIN1s. Of 18 CIN2s, 5 cases progressed to CIN3 within 9 to 30 months. Lesions persisted in $5 \mathrm{CIN} 2 \mathrm{~s}$ for 13 to 47 months and regressed within 3 to 6 months in 8 CIN2s. In each case, paraffin-embedded blocks obtained at the initial diagnosis and every follow-up period were subjected for analysis.

\section{Laser Capture Microdissection and DNA Extraction}

Formalin-fixed, paraffin-embedded sections were cut at $4 \mu \mathrm{m}$ at five serial levels, placed on glass slides, and stained with hematoxylin-eosin. After hematoxylineosin staining, the sections were dehydrated in graded alcohols, deparaffinized in xylene, and air dried. Five sections of each sample were microdissected using the LM200 LCM system (Arcturus Engineering, Santa Clara, California). DNA was extracted from the microdissected samples by protease $\mathrm{K}$ digestion, followed by phenol/chloroform extraction as previously described (Wada et al, 2000). Corresponding normal epithelial cells were extracted and used as controls for clonality analysis.

\section{Analysis of X Chromosome Inactivation by PCR Amplification}

The human androgen receptor (HUMARA), which is mapped to Xcen-q13, contains an in-frame CAG trimeric repeat encoding 11 to 31 glycine residues in exon 1 that is polymorphic in $90 \%$ of cases. The methylation status of $H$ hal sites, which are located approximately $100 \mathrm{bp}$ upstream of the trinucleotide repeat element, correlates with $X$ chromosome inactivation (Allen et al, 1992). The patterns of $X$ chromosome inactivation of the lesions were analyzed by methylation-sensitive restriction endonuclease Hhal 
treatment of the extracted DNA, followed by PCR amplification of the human androgen receptor target as previously reported (Sun et al, 2002). Briefly, DNA extracted from paraffin-embedded archival sections was digested with $2 \mathrm{U}$ of Hhal in $10 \mu$ of digestion buffer at $37^{\circ} \mathrm{C}$ for 12 hours. After terminating digestion by incubation at $95^{\circ} \mathrm{C}$ for 10 minutes, the DNAs were re-extracted with phenol/chloroform, precipitated with ethanol, and used as a PCR template. Undigested DNA was retained as a control.

Nested PCR was performed to increase the sensitivity of amplification using previously published primers (Sun et al, 2002). Conditions for PCR amplification were as previously described (Sun et al, 2002). One microliter of the amplified product was subjected to a second round of PCR amplification under the conditions described above. The PCR products were processed on the ABI PRISM 310 Genetic Analyzer (Perkin-Elmer Corporation, Foster City, California), and the results were analyzed using ABI PRISM GeneScan Analysis Software (Version 2.1). PCR amplification was also performed on histologically normal endometrial epithelial cells or stromal tissue from each case. All samples were run in duplicate to avoid misinterpretation or laboratory error and to demonstrate reproducibility.

\section{Detection of HPV by PCR-RFLP}

The presence of HPV was examined by PCR to generate amplified DNA fragments within the ORFs of E6 and E7 using consensus sequence primers as follow: pU-1M, 5'-TGTCAAAAACCGTTGTGTCC-3'; pU-31B, 5'-TGCTAATTCGGTGCTACCTG-3'; and pU2R, 5'-GAGCTGTCGCTTAATTGCTC-3', as described by Fujinaga et al (1991). Low-risk HPVs (types 6 and 11) were detected by amplification with primers $p U-2 R$ and pU31B. High-risk HPVs (types 16, 18, 31, 33, 35, $52 \mathrm{~b}$, and 58) were amplified using primers $\mathrm{pU}-2 \mathrm{R}$ and $\mathrm{pU}-1 \mathrm{M}$. Five microliters of the PCR products were digested with $4 \mathrm{U}$ of restriction enzymes Rsal, Avall, and Sau3Al independently for 3 hours, according to procedures described by the manufacturer (TaKaRa, Tokyo, Japan). The digestion products were fractionated on $4 \%$ nondenaturing agarose gels, stained with ethidium bromide, and photographed.

\section{Analysis of HPV-16 Physical Status by Real-Time PCR}

The physical status of HPV-16 in CIN was examined by real-time PCR amplification of the E2 and E6 ORFs. Primers for E2 ORF were designed to amplify the E2 hinge region, which is usually deleted upon viral integration into the host genome in cervical carcinoma (Kalantari et al, 1998). The primers were as follows: sense primer, 5'-ACGACTATCCAGCGACCAAGAT-3' (nucleotide position 3503-3524), and antisense primer, 5'-CCAATGCCATGTAGACGACACT-3' (nucleotide position 3717-3738) for E2 ORF; and sense primer, 5'-GAACTGCAATGTTTCAGGACCC-3' (nucleotide position 26-47) and antisense primer, 5'ATATACCTCACGTCGCAGTAACTGTT-3' (nucleotide position 210-232) for E6 ORF (Park et al, 1997). TaqMan probes were 5'-FAM-CCTGCCACACCACTAAGTTGTTGCACA-TAMRA-3' for E2 ORF and 5'-FAMCAGGAGCGACCCAGAAAGTTACCACAGTTAMRA-3' for E6 ORF. Fifty microliters of the PCR reaction buffer consisted of $5 \mu \mathrm{l}$ of template DNA, $5 \mu \mathrm{m}$ of each sense and antisense primer, $2 \mu \mathrm{M}$ of TaqMan probe, and $25 \mu \mathrm{l}$ of the TaqMan Universal PCR Master Mix (Applied Biosystems, Foster City, California). The amplification conditions were 2 minutes at $50^{\circ} \mathrm{C}, 10$ minutes at $95^{\circ} \mathrm{C}$, followed by a two-step cycle of $95^{\circ} \mathrm{C}$ for 15 seconds and $60^{\circ} \mathrm{C}$ for 60 seconds for a total of 50 cycles. The cutoff value for the ratio of E2 to E6 copy numbers was set at 0.5 on the basis of the reliability of the real-time PCR. E2 and E6 ORFs of HPV-16 were quantified with the ABI PRISM 7700 Sequence Detection System (Applied Biosystems). Cloned HPV-16 DNA that contained the whole genome of HPV-16 and PZE67 that contained E6-E7 ORF of HPV-16 (Yutsudo et al, 1988), both kindly provided by Dr. M. Yutsudo, were used as controls for the episomal and integrated forms, respectively.

\section{Statistical Analysis}

Statistical assessment of the data was performed using the two-tailed Fisher exact test.

\section{Acknowledgements}

We thank Dr. M. Yutsudo, Department of Tumor Virology, Research Institute for Microbial Disease, Osaka University, for providing the HPV plasmid.

\section{References}

Allen RC, Zoghbi HY, Moseley AB, Rosenblatt HM, and Belmont JW (1992). Methylation of Hpall and Hhal sites near the polymorphic CAG repeat in the human androgenreceptor gene correlates with $X$ chromosome inactivation. Am J Hum Genet 51:1229-1239.

Bergeron C, Barrasso R, Beaudenon S, Flamant P, Croissant O, and Orth G (1992). Human papillomaviruses associated with cervical intraepithelial neoplasia. Am J Surg Pathol 16:641-649.

Das BC, Sharma JK, Gopalakrishna V, and Luthra UK (1992). Analysis by polymerase chain reaction of the physical state of human papillomavirus type 16 DNA in cervical preneoplastic and neoplastic lesions. J Gen Virol 73:2327-2336.

Enomoto T, Fujita M, Inoue M, Tanizawa O, Nomura T, and Shroyer KR (1994). Analysis of clonality by amplification of short tandem repeats: Carcinomas of the female reproductive tract. Diagn Mol Pathol 3:292-297.

Enomoto T, Haba T, Fujita M, Hamada T, Yoshino K, Nakashima R, Wada H, Kurachi H, Wakasa K, Sakurai M, Murata Y, and Shroyer KR (1997). Clonal analysis of highgrade squamous intra-epithelial lesions of the uterine cervix. Int J Cancer 73:339-344.

Fialkow PJ (1976). Clonal origin of human tumors. Biochem Biophys Acta 485:283- 321.

Fujinaga Y, Shimada M, Okazawa K, Fukushima M, Kato I, and Fujinaga K (1991). Simultaneous detection and typing of genital human papillomavirus DNA using the polymerase chain reaction. J Gen Virol 72:1039-1044. 
Iwasaka T, Yokoyama M, and Matsuo N (1994). In situ hybridization analysis of human papillomavirus DNA in cervical intraepithelial neoplasia. Cervix Low Female Genital Tract 12:55-59.

Kalantari M, Karlsen F, Kristensen G, Holm R, Hagmar B, and Johanson $B$ (1998). Disruption of the $E 1$ and $E 2$ reading frames of HPV16 in cervical carcinoma is associated with poor prognosis. Int J Gynecol Pathol 17:146-153.

Kataja V, Syrjanen S, Mantyjarvi R, Yliskoski M, Saarikoski S, and Syrjanen K (1992). Prognostic factors in cervical human papillomavirus infections. Sex Transm Dis 19:154-160.

Mandel JL, Manaco AP, Nelson DL, Schlessinger D, and Willard H (1992). Genome analysis and the human X chromosome. Science 258(5079):103-109.

Murthy NS, Sardana S, Narang N, Agarwal SS, Sharma S, and Das DK (1996). Biological behaviour of moderate dysplasia. Indian J Cancer 33(1):24-30.

Murthy NS, Sehgal A, Satyanarayana L, Das DK, Singh V, Das BC, Gupta MM, and Luthra UK (1990). Risk factors related to biological behaviour of precancerous lesions of the uterine cervix. Br J Cancer 61(5):732-736.

Nagao S, Yoshinouchi M, Miyagi Y, Hongo A, Kodama J, Itoh $S$, and Kudo T (2002). Rapid and sensitive detection of physical status of human papillomavirus type 16 DNA by quantitative real-time PCR. J Clin Microbiol 40:863-867.

Nasiell K, Nasiell M, and Vaclavinkova V (1983). Behavior of moderate cervical dysplasia during long-term follow-up. Obstet Gynecol 61(5):609-614.

Nasiell K, Roger V, and Nasiell M (1986). Behavior of mild cervical dysplasia during long-term follow-up. Obstet Gynecol 67(5):665-669.

Noda K, Yajima A, Higashiiwai H, Sato A, and Terashima K (1976). Histopathologic criterion of dysplasia of the uterine cervix and its biological nature. Acta Cytologia 20:224-228.
Peitsaro P, Johansson B, and Syrjanen S (2002). Integrated human papillomavirus type 16 is frequently found in cervical cancer precursors as demonstrated by a novel quantitative real-time PCR technique. J Clin Microbiol 40:886-891.

Park JS, Hawing ES, Park SN, An HK, Um SJ, Kim CJ, Kim SJ, and Naming SE (1997). Physical status and expression of HPV genes in cervical cancers. Gynecol Oncol 65:121-129.

Park TW, Richard RM, Sun XW, and Wright TC Jr (1996). Association between human papillomavirus type and clonal status of cervical squamous intraepithelial lesions. J Natl Cancer Inst 88:355-358.

Riggs AD and Pfeifer GP (1992). X-chromosome inactivation and cell memory. Trends Genet 8:169-174.

Sun H, Enomoto T, Shroyer KR, Ozaki K, Fujita M, Ueda Y, Nakashima R, Kuragaki C, Ueda G, and Murata Y (2002). Clonal analysis and mutations in the PTEN and the K-ras genes in endometrial hyperplasia. Diagn Mol Pathol 11:204211.

Wada H, Enomoto T, Yoshino K, Ozaki K, Kurachi H, Nomura T, Murata Y, Kim N, Weinrich S, Lee CE, Lopez UD, and Shroyer KR (2000). Immunohistochemical localization of telomerase hTERT protein and analysis of clonality in multifocal vulvar intraepithelial neoplasia. Am J Clin Pathol 114(3):371379 .

Yutsudo M, Okamoto Y, and Hakura A (1988). Functional dissociation of transforming genes of human papillomavirus type 16. Virology 166:594-597.

zur Hausen $H$ (1991). Human papillomaviruses in the pathogenesis of anogenital cancer. Virology 184:9-13. 